\title{
Effect of Radiation on Magnetic Flow over a Moving Vertical Plate in a Porous Medium with Heat Generation
}

\author{
K.Gangadhar ${ }^{1}$, B. Hema Sundar Raju ${ }^{2}$, M. Venkata Subba Rao ${ }^{3}$ and M. \\ Sreedhar $\mathrm{Babu}^{4}$ \\ ${ }^{1}$ Department of Mathematics, Acharya Nagarjuna University, Ongole, Andhra Pradesh -523001, India \\ ${ }^{2,3}$ Department of Mathematics, Vignan University, Guntur, Andhra Pradesh-522213, India \\ ${ }^{4}$ Department of Mathematics, Yogi Vemana University, Kadapa, A. P, India
}

\begin{abstract}
Steady laminar natural convection of MHD flow over a semi-infinite moving vertical plate in the presence of internal heat generation and a convective surface boundary condition in a porous medium is examined in this paper. It is assumed that the left surface of the plate is in contact with a hot fluid while the cold fluid on the right surface of the plate contains a heat source that decays exponentially with the classical similarity variable. The governing non-linear partial differential equations have been transformed by a similarity transformation into a system of ordinary differential equations, which are solved numerically by using the shooting techniques with the forth order Runga-Kutta method. The effects of physical parameters on the dimensionless velocity and temperature profiles are depicted graphically and analyzed in detail. Finally, numerical values of physical quantities, such as the local skin-friction coefficient and the local Nusselt number are presented in tabular form. It is observed that the velocity and thermal boundary layer thicknesses decrease with an increase in the intensity of radiation parameter.
\end{abstract}

Key words: moving vertical plate, internal heat generation, radiation, local Biot number, MHD, Porous medium.

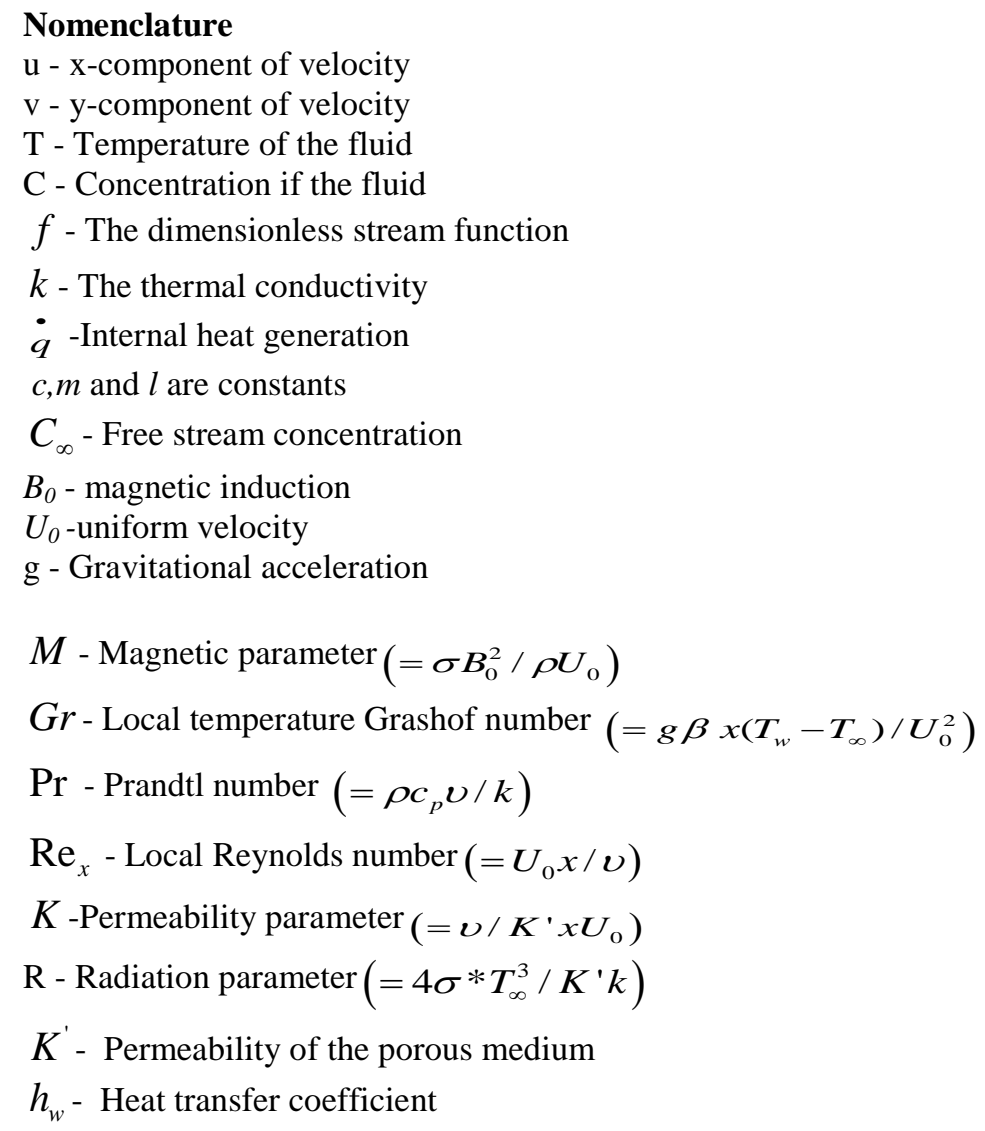




\section{Greek Symbols}

$v$ - Fluid kinematics viscosity

$\rho$ - Density

$\sigma$ - Electric conductivity of the fluid

$\beta$ - Coefficients of thermal expansion

$\gamma$-liocal Biot number $\left(=\left\{h_{w}\left(v x / U_{0}\right)^{\frac{1}{2}}\right\} / k\right)$

$\eta$ - Similarity variable

$\theta(\eta)$ - Dimensionless temperature

$\psi$ - Streem function

\section{Introduction}

Boundary layer flows with internal heat generation past a vertical plate continues to receive considerable attention because of its many practical applications in a broad spectrum of engineering systems like geothermal reservoirs, cooling of nuclear reactors, thermal insulation, combustion chamber, rocket engine, etc. Many principal past studies concerning natural convection flows over a semi-infinite vertical plate immersed in an ambient fluid have been found in the literature (Baksr (1976), Makinde (2009), Paresh Vyas\&Ashutosh Ranjan (2010)). In many cases, when natural convection takes place from a vertical flat plate, the temperature is not isothermal and these problems may admit similarity solutions. Crepeau et al. (1997) reported a local similarity solution for a natural convection over a vertical plate with isothermal surface temperature and internal heat generation. Kao (1976) investigated free convection from vertical plates with sinusoidal temperature variation and constant transpiration.

The effect of radiation on free convection flow of fluid with variable viscosity from a porous plate is discussed Anwar Hossain et al (2001). The fluid considered in that paper is an optically dense viscous incompressible fluid of linearly varying temperature dependent viscosity. Salem (2006) discussed coupled heat and mass transfer in Darcy-Forchheimer Mixed convection from a vertical flat plate embedded in a fluid saturated porous medium under the effects of radiation and viscous dissipation. Paresh Vyas \& Ashutosh Ranjan (2010) discussed the dissipative MHD boundary- layer flow in a porous medium over a sheet stretching nonlinearly in the presence of radiation. Seddeek and Almushigeh (2010) studied the Effects of radiation and variable viscosity on MHD free convective flow and mass transfer over a stretching sheet with chemical reaction. The idea of using a convective boundary condition was recently introduced by Aziz (2009) to study the classical boundary layer flow over a flat plate. Soret and Dufour effects on hydro magnetic Heat and Mass Transfer over a Vertical Plate with a Convective Surface Boundary Condition and Chemical reaction were studied by Gangadhar (2013). Makinde et al. (2010) reported a local similarity solution for the effect of buoyancy forces on thermal boundary layer over a flat plate with a convective boundary condition. Their result also revealed that the buoyancy effects tend to reduce the thermal boundary layer thickness. Gangadhar and Bhaskar Reddy (2013) has analyzed by chemically reacting MHD boundary layer flow of heat and mass transfer over a moving vertical plate in a porous medium with suction. Anwar Beg et al (2011) was discussed by the Mathematical modeling on oscillatory MHD couette flow in a rotating highly permeable medium permeated by an oblique magnetic field.

The oscillatory flow past a moving plate in the presence of free convection and radiation was studied by Mansour (1990). The unsteady flow past a moving plate in the presence of free convection and radiation was studied by Raptis and Perdikis (1999). Das et al. (1996) analyzed the radiation effects on the flow past an impulsively started infinite isothermal vertical plate. Abd-El-Naby et al. (2003) studied the effects of radiation on an unsteady free convective flow past a semi-infinite vertical plate with a variable surface temperature. Chamkha et al. (2001) studied the effect of radiation on free convective flow past a semi-infinite vertical plate with mass transfer. Finite difference analysis of radiative free convection flow past an impulsively started vertical plate with variable heat and mass flux was studied by Ramachandra Prasad et al (2007).

In this paper, the similarity method is employed to investigate the effect of an exponentially decaying internal heat generation on a MHD boundary layer flow over a moving vertical plate in a porous medium with a convective boundary condition. The numerical computation for the resulting nonlinear differential equation is performed using a fourth-order Runge-Kutta method with shooting technique. Pertinent results are displayed graphically and discussed quantitatively. 


\section{Mathematical Analysis}

We consider the steady MHD laminar incompressible natural convection boundary layer flows over the right surface of a vertical flat plate moving with uniform velocity $U_{0}$ in contact with a quiescence cold fluid at temperature $T_{\infty}$. The cold fluid on the right surface of the plate generates heat internally at the volumetric rate $\dot{q}$. The left surface of the plate is heated by convection from a hot fluid at temperature $T_{w}$ which provides a heat transfer coefficient $h_{w}$. Under the Boussinesq for fluid density variation, the continuity, momentum, and energy equations describing the flow can be written as:

$$
\begin{aligned}
& \frac{\partial u}{\partial x}+\frac{\partial v}{\partial y}=0 \\
& u \frac{\partial u}{\partial x}+v \frac{\partial u}{\partial y}=v \frac{\partial^{2} u}{\partial y^{2}}-\frac{\sigma B_{0}^{2}}{\rho} u+g \beta\left(T-T_{\infty}\right)-\frac{v}{K} u \\
& \rho c_{p}\left(u \frac{\partial T}{\partial x}+v \frac{\partial T}{\partial y}\right)=k \frac{\partial^{2} T}{\partial y^{2}}-\frac{\partial q_{r}}{\partial y}+\dot{q}
\end{aligned}
$$

Where $u$ and $v$ are the $x$ (along the plate) and the $y$ (normal to the plate) components of the velocities, respectively, $T$ is the temperature, $v$ - the kinematics viscosity of the fluid, $\rho$ - the fluid density, $c_{p}$ - the specific heat at constant pressure, $B_{0}$ - the magnetic induction, $K^{\prime}$ - the permeability of the porous medium, $k$ the thermal conductivity of the fluid, and $\beta$ - the thermal expansion coefficient.

The boundary conditions at the plate surface and far into the cold fluid may be written as:

$$
\begin{aligned}
& u(x, 0)=U_{0}, v(x, 0)=0,-k \frac{\partial T}{\partial y}(x, 0)=h_{w}\left[T_{w}-T(x, 0)\right] \\
& u(x, \infty)=0, T(x, \infty)=T_{\infty}
\end{aligned}
$$

By using the Rosseland approximation (Brewster (1992)), the radiative heat flux $q_{r}$ is given by

$$
q_{r}=-\frac{4 \sigma^{*}}{3 K^{\prime}} \frac{\partial T^{4}}{\partial y}
$$

where $\sigma^{*}$ is the Stefan-Boltzmann constant and $K^{\prime}$ - the mean absorption coefficient. It should be noted that by using the Rosseland approximation, the present analysis is limited to optically thick fluids. If temperature differences within the flow are sufficiently small, then Equation (7) can be linearized by expanding $T^{4}$ into the Taylor series about $T_{\infty}$, which after neglecting higher order terms takes the form

$$
T^{4} \cong 4 T_{\infty}^{3} T-3 T_{\infty}^{4}
$$

In view of equations (6) and (7), equation (3) reduces to

$$
u \frac{\partial T}{\partial x}+v \frac{\partial T}{\partial y}=\alpha\left(1+\frac{16 \sigma^{*} T_{\infty}^{3}}{3 K^{\prime} k}\right) \frac{\partial^{2} T}{\partial y^{2}}+\frac{1}{\rho c_{p}} \dot{q}
$$

The continuity equation (1) is satisfied by the Cauchy Riemann equations

$$
u=\frac{\partial \psi}{\partial y} \text { and } v=-\frac{\partial \psi}{\partial x}
$$

Introducing a similarity variable $\eta$ and a dimensionless stream function $f(\eta)$ and temperature $\theta(\eta)$ as:

$$
\eta=\frac{y}{x} \sqrt{\operatorname{Re}_{x}}, u=U_{0} f^{\prime}, v=\frac{v}{2 x} \sqrt{\operatorname{Re}_{x}}\left(\mu f^{\prime}-f\right), \theta(\eta)=\frac{T-T_{\infty}}{T_{w}-T_{\infty}}, \lambda_{x}=\frac{\dot{q} x^{2} e^{\eta}}{k \operatorname{Re}_{x}\left(T_{w}-T_{\infty}\right)}
$$

Where the prime symbol denotes differentiation with respect to $\eta$ and $\operatorname{Re}_{x}=\frac{U_{0} x}{v}$ is the local

Reynolds number. Substituting eq.(10) into the eqs. (2), (8), (4) and (5), we obtain:

$$
\begin{aligned}
& f^{\prime \prime \prime}+\frac{1}{2} f f^{\prime \prime}+G r \theta-(M+K) f^{\prime}=0 \\
& \left(\frac{3 R+4}{3 R \operatorname{Pr}}\right) \theta^{\prime \prime}+\frac{1}{2} f \theta^{\prime}+\lambda_{x} e^{-\eta}=0 \\
& f(0)=0, f^{\prime}(0)=1, \theta^{\prime}(0)=\gamma[\theta(0)-1] \\
& f^{\prime}(\infty)=0, \theta(\infty)=0
\end{aligned}
$$


The local internal heat generation parameter $\lambda_{x}$ is defined such that the internal heat generation decays exponentially with the similarity variable $\eta$ as stipulated in highlighted in eq. (12). This type of model can be used in mixtures where a radioactive material is surrounded by inert alloys and in the electromagnetic heating of materials (Jaluria (1980), Sahin(1992)). Moreover, eqs.(11)-(13) will definitely produce a local similarity solution for the problem. In order to have a true similarity solution, the parameters $M, G r, \lambda_{x}, \gamma, R$ and $P r$ must be constant. This condition will be satisfied if the heat transfer coefficient $h_{w}$ is proportional to $x^{-\frac{1}{2}}$, the thermal expansion coefficient $\beta$ and internal heat generation $\dot{q}$ are proportional to $x^{-1}$. Hence, we assume

$$
h_{w}=c x^{-\frac{1}{2}}, \beta=m x^{-1}, \dot{q}=l x^{-1} e^{-\eta}
$$

Where $c, m$ and $l$ are constants but have the appropriate dimensions. Substituting eq. (14) into the parameters Gr, $\lambda_{x}$ and $\gamma$.

We obtain, $\gamma=\frac{c}{k} \sqrt{\frac{\nu}{U_{0}}}, G r=\frac{m g\left(T_{w}-T_{\infty}\right)}{U_{0}^{2}}$ and $\lambda_{x}=\frac{l v}{k U_{0}\left(T_{w}-T_{\infty}\right)}$

The coupled nonlinear boundary value problem represented by eqs.(11)-(13) have been solved numerically using the shooting techniques with the forth order Runga-Kutta method. From the numerical computation, the plate surface temperature, the local skin-friction coefficient and the local Nusselt number which are, respectively, proportional to $\theta(0), f^{\prime \prime}(0)$ and $-\theta^{\prime}(0)$ are worked out and their numerical values are presented in a tabular form.

\section{Results And Discussion}

The positive values of local Grashof number $G r>0$ is utilised in our computations. This corresponds to the cooling problem with respect to application. The cooling problem is often encountered in engineering applications; for example in the cooling of electronic components and nuclear reactors. Table $1 \& 2$ illustrates the values of local skin friction coefficient, local Nusselt number and plate surface temperature for different values of parameters embedded in the system. Physically, positive sign of skin friction implies that the fluid exerts a drag force on the right surface of the plate and the negative sign implies the opposite. Both local skin friction and local Nusselt number coefficients increases with an increase in local Biot number, although the presence of back flow of heat into the plate is noticed for $\operatorname{Pr}=0.72$ (air) since $\theta^{\prime}(0)$ is positive except for the case of $\operatorname{Pr}=$ 7.1 (water) when $\theta^{\prime}(0)$ is negative and the heat flows out from the left surface of the plate to its right surface. Similar trend is observed as the internal heat generation increases $i . e$. as increases from 1 to 10 with an increase in both local skin friction and local Nusselt number coefficients. As $G r$ increases from 0.1 to 2, the local skin friction increases because of the increased strength of the buoyancy forces but the back heat flow into the plate (caused by the internal heat generation) is reduced. The local skin friction coefficient decreases and local Nusselt number coefficient increases with an increase in both permeability parameter and magnetic field parameter. As radiation parameter increases from 0 to 10, the local skin-friction and local Nusselt number increases. Makinde (2011) found them in excellent agreement as demonstrated in table 1.

Figures 1-14 depict the effects of various thermophysical parameters on the fluid velocity and temperature profiles. Generally, the fluid velocity increases gradually away from the plate, attain its peak value within the boundary layer and then decreases to the free stream zero value satisfying the boundary conditions. The fluid temperature is highest near the plate surface and decreases exponentially to zero value far away from the plate.

It is interesting however to note that the velocity boundary layer thickness decreases while the thermal boundary layer thickness increases with an increase in the value of magnetic parameter $(M)$ (see figs. 1-2). In figs. 3-4 we observed that both velocity and thermal boundary layer thicknesses decrease with an increase in the intensity of local Biot number $(\gamma)$ due to convective heat transfer the plate surface. In figs. 5 and 6 we observed that both velocity and thermal boundary layer thicknesses increase with an increase in the Prandtl number $(P r)$. In figs. $7 \& 8$ we observed that the velocity boundary layer thickness increases while the thermal boundary layer thickness decreases with an increase in the value of local Grashof number $(G r)$ due to buoyancy effect. An increase in the exponentially decaying internal heat generation $\left(\lambda_{x}\right)$ causes a further increase in both velocity and thermal boundary layer thicknesses as shown in figs. $9 \& 10$. In figs. $11 \& 12$ we observed that the velocity boundary layer thickness decreases while the thermal boundary layer thickness increases with an increase in the value of permeability parameter $(K)$. In figs. 13 and 14 we observed that both velocity and thermal boundary layer thicknesses decrease with an increase in the intensity of radiation parameter $(R)$. 


\section{Conclusions}

In this paper we have studied numerically the effects of radiation and internal heat generation on MHD boundary layer flow over a vertical plate in a porous medium with a convective surface boundary condition. From the present study we have found among others that the internal heat generation prevents the flow of heat from the left surface to the right surface of the plate unless the local Grashof number is strong enough to convert away both the internally generated heat in the fluid. Both local skin friction and local Nusselt number coefficients increases with an increase in local Biot number. The local skin friction coefficient decreases and local Nusselt number coefficient increases with an increase in both permeability parameter and magnetic field parameter. Both local skin friction and local Nusselt number coefficients decreases with an increase in radiation parameter.

\section{References}

[1] Abd-El-Naby M.A., Elasayed M.E., Elbarbary, Nader Y. and Abdelzem .(2003), Finite difference solution of radiation effects on MHD free convection flow over a mvertical plate with variable surface temperature, J.Appl .Math., Vol.2, pp. 65-86.

[2] Anwar Beg, O,Ghosh, S.K and Narahari, M.,(2011), Mathematical modeling on oscillatory MHD couette flow in a rotating highly permeable medium permeated by an oblique magnetic field, chemical engineering communications, Vol.198, pp.235-254.

[3] Anwar Hossain.M, Khalil Khanafer, Kambiz Vafai (2001), "The effect of radiation on free convection flow of fluid with variable viscosity from a porous vertical plate", International Journal of Thermal Science, vol.40, 2001,pp. 115-124.

[4] Aziz A. (2009), A Similarity Solution forLaminar Thermal Boundary Layer over a Flat Plate with a Convective Surface Boundary Condition, Communicatons in Nonlinear Science and Numerical Simulations, 14 (2009), 4, pp. 1064-1068.

[5] Baker L., Faw, R. E., Kalacki, F. A. (1976),Postaccident Heat Removal - Part I: Heat Transfer within an Internally Heated, Nonboiling Liquid Layer, Nuclear Science and Engineering, 61 (1976), 2, pp. 222-230.

[6] Brewster M.Q., (1992), Thermal radiativetransfer and properties, John Wiley \& Sons, New York.

[7] Chamkha A.J., Takhar H.S. and Soundalgekar V.M. (2001), Radiation effects on freeconvection flow past a semi-infinite vertical plate with mass transfer, Che. Engg.J., Vol.84, pp. 335-342

[8] Crepeau J. C., Clarksean, R.(1997), Similarity Solutions of Natural Convection with Internal Heat Generation, Trans. ASME, Journal of Heat Transfer, 119 (1997), 5, pp. 184-185

[9] Das U.N., Deka R. and Soundalgekar V.M.,(1996), Radiation effects on flow past an impulsively started vertical plate-an exact solutions, J. Theo .Appl. Fluid Mech., Vol. 1(2), pp.111-115.

[10] Gangadhar, K., (2013), Soret and DufourEffects on Hydro Magnetic Heat and Mass Transfer over a Vertical Plate with a Convective Surface Boundary Condition and Chemical Reaction, Journal of Applied Fluid Mechanics, Vol. 6, No. 1, pp. 95-105.

[11] Gangadhar, K. and Bhaskar Reddy, N., (2013),Chemically Reacting MHD Boundary Layer Flow of Heat and Mass Transfer over a Moving Vertical Plate in a Porous Medium with Suction, Journal of Applied Fluid Mechanics, Vol. 6, No. 1, pp. 107-114.

[12] Jaluria, Y. (1980), Natural Convection Heat andMass Transfer, Pergamon Press, New York, USA.

[13] Kao T. T.(1976), Locally Nonsimilar Solutionfor Laminar Free Convection Adjacent to a Vertical Wall, Trans. ASME, Journal of Heat Transfer, 98 , pp. 321-322.

[14] Makinde.O.D.(2011), "Similarity solution fornatural convection from a moving vertical plate with internal heat generation and a convective boundary condition. Thermal science,Vol.15,suppl.1,pp.S137-S143.

[15] Makinde O. D. (2009), On MHD Boundary-Layer Flow and Mass Transfer Past a Vertical Plate in a Porous Medium with Constant Heat Flux, International Journal of Numerical Methods for Heat \& Fluid Flow, 19 (2009), 3/4, pp. 546-554

[16] Makinde O. D., Olanrewaju, P. O. (2010),Buoyancy Effects on Thermal Boundary Layer over a Vertical Plate with a Convective Surface Boundary Condition, Trans. ASME, Journal of Fluid Engineering, 132 (2010), 1-4, pp. 044502.

[17] Mansour M.H. (1990), Radiative and free convection effects on the oscillatory flow past a vertical plate, Astrophysics and space science, Vol. 166, pp.26-75.

[18] Ostrach S (1953), An Analysis of Laminar Free Convection Flow and Heat Transfer about a Flat Plate Parallel to the Direction of the Generating Body Force, NACA Technical Report 1111, 1953.

[19] Paresh Vyas\&Ashutosh Ranjan (2010).

[20] 'Discussed the dissipative MHD boundary- layer flow in a porous medium over a sheet stretching nonlinearly in the presence of radiation' Applied Mathematical Sciences, Vol. 4, 2010, no. 63, 3133 - 3142.

[21] Ramachandra Prasad V, Bhaskar Reddy N and Muthucumaraswamy R (2007), Finite difference analysis of radiative free convection flow past an impulsively started vertical plate with variable heat and mass flux, J. Appl. Theo. Mech., (in press). Raptis A. and Perdikis C. (1999), Radiation and free convection flow past a moving plate, Appl. Mech. Eng., Vol.14, pp. 817-821.

[22] Salem A.M, (2006) "Coupled heat and mass transfer in Darcy-Forchheimer Mixed convection from a vertical flat plate embedded in a fluid saturated porous medium under the effects of radiation and viscous dissipation", Journal of the Korean Physical Society, 48(3), 2006, pp.109-113.

[23] Seddeek M. A. and A. A. Almushigeh (2010). 'Effects of radiation and variable viscosity on MHD free convective flow and mass transfer over a stretching sheet with chemical reaction'. Applications and Applied Mathematics. Vo. 5, No.1, pp.181-197.

[24] Sahin A. Z (1992), Transient Heat Conductionin Semi-Infinite Solid with Spatially Decaying Exponential Heat Generation. International Communication in Heat Mass Transfer, 19 (1992), 3, pp. 349-358. 


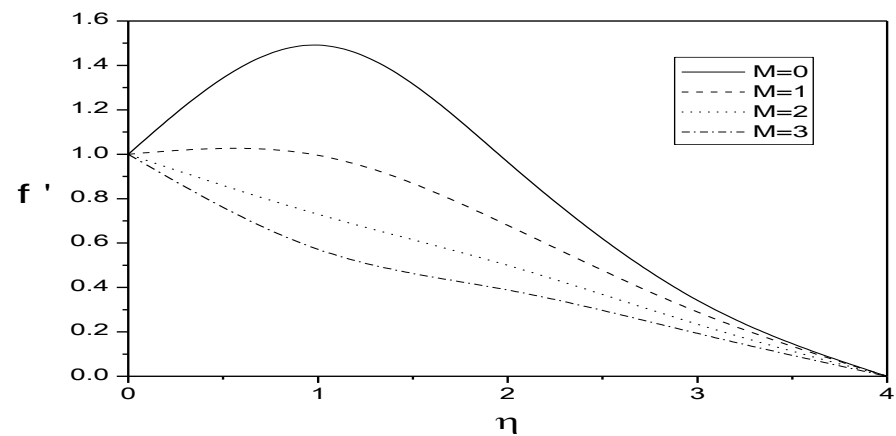

Fig.1 Variation of the velocity component $f^{\prime}$ with $M$ for $G r=\gamma=K=0.1, \lambda_{x}=10, R=1, \operatorname{Pr}=0.72$.

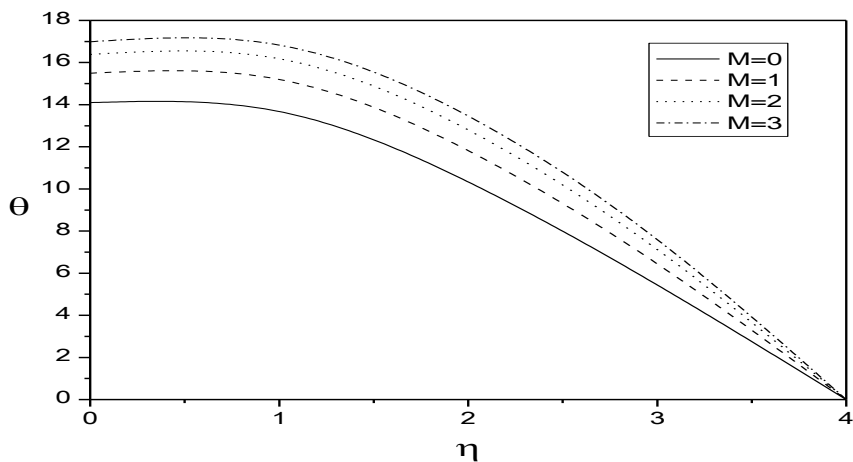

Fig.2 Variation of the temperature $\theta$ with $M$ for $G r=\gamma=K=0.1, \lambda_{x}=10, R=1, \operatorname{Pr}=0.72$.

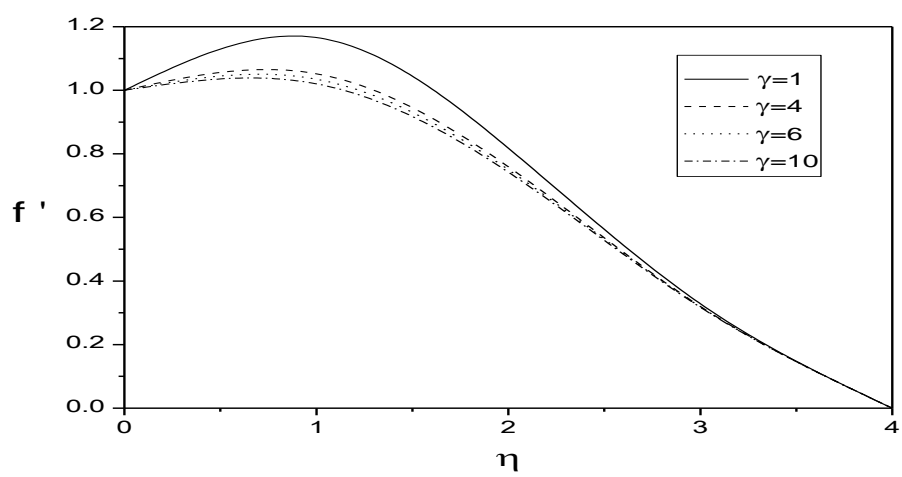

Fig.3 Variation of the velocity component $f^{\prime}$ with $\gamma$ for $G r=M=K=0.1, \lambda_{x}=10, R=1, \quad \operatorname{Pr}=0.72$.

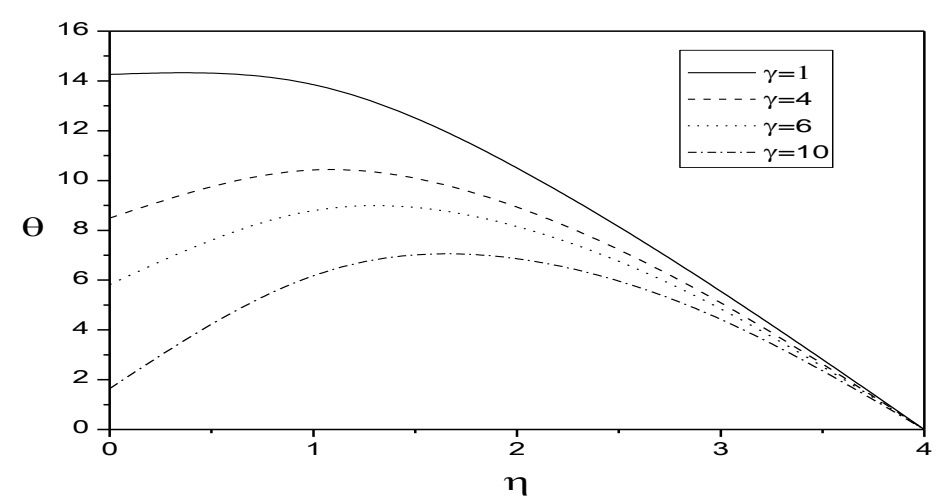

Fig.4 Variation of the temparature $\theta$ with $\gamma$ for $G r=M=K=0.1, \lambda_{x}=10, R=1, \operatorname{Pr}=0.72$. 


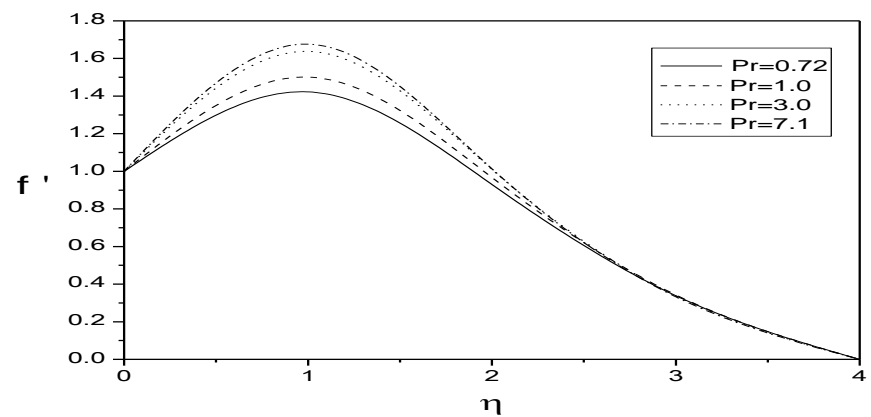

Fig.5 Variation of the velocity component $f^{\prime}$ with $\operatorname{Pr}$ for $G r=\gamma=K=M=0.1, R=1, \lambda_{x}=1$.

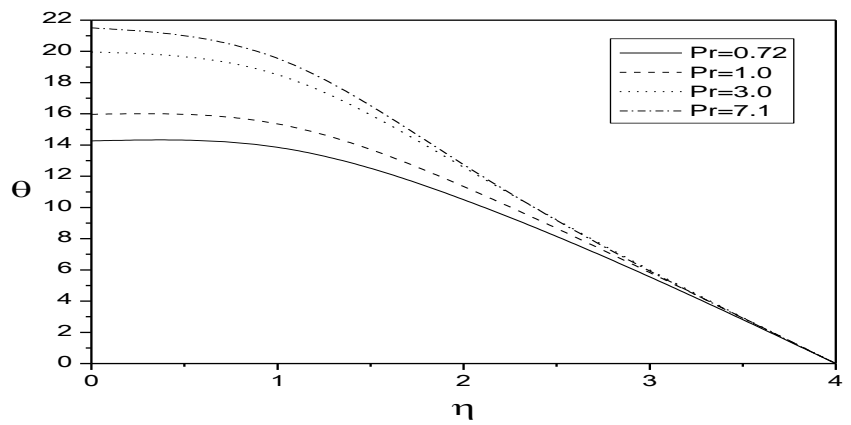

Fig.6 Variation of the temperature $\theta$ with $\operatorname{Pr}$ for $G r=\gamma=K=M=0.1, R=1, \lambda_{x}=10$.

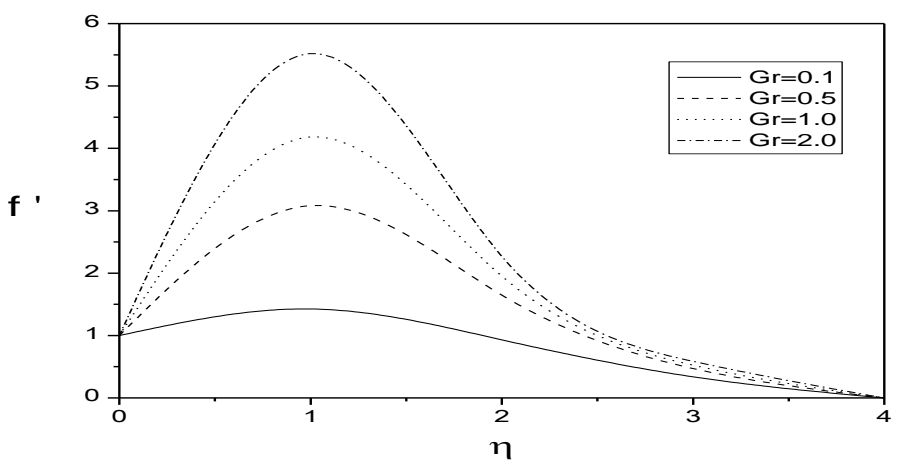

Fig.7 Variation of the velocity component $f^{\prime}$ with $\boldsymbol{G r}$ for $M=\gamma=K=0.1, \lambda_{x}=10, R=1, \operatorname{Pr}=0.72$.

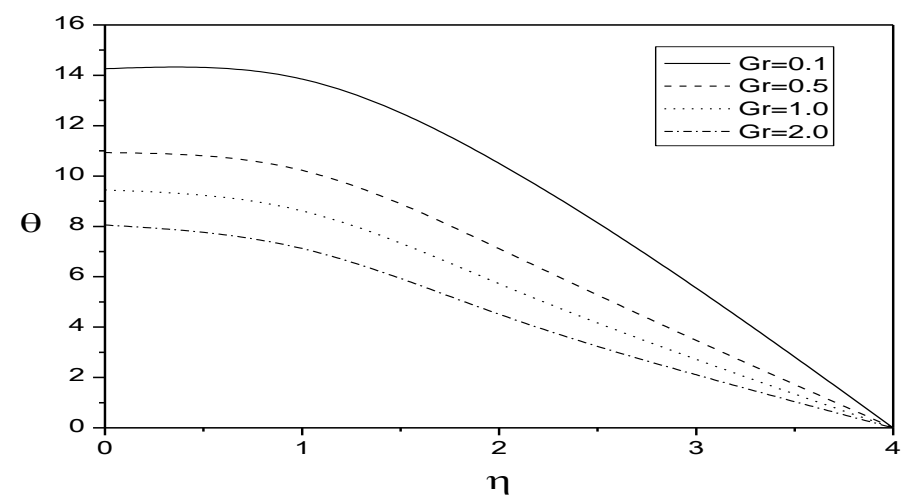

Fig.8 Variation of the temperature $\boldsymbol{\theta}$ with $\boldsymbol{G r}$ for $M=\gamma=K=0.1, \lambda_{x}=10, R=1, \operatorname{Pr}=0.72$. 


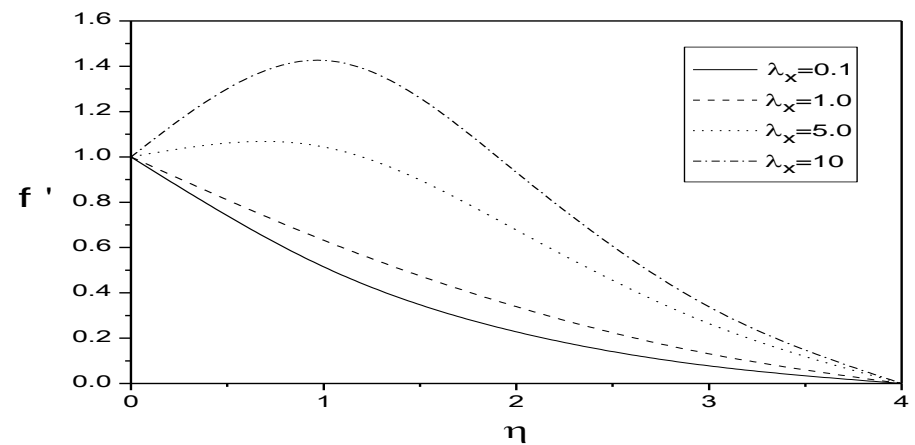

Fig.9 Variation of the velocity component $f^{\prime}$ with $\lambda_{x}$ for $G r=\gamma=K=M=0.1, R=1, \operatorname{Pr}=0.72$.

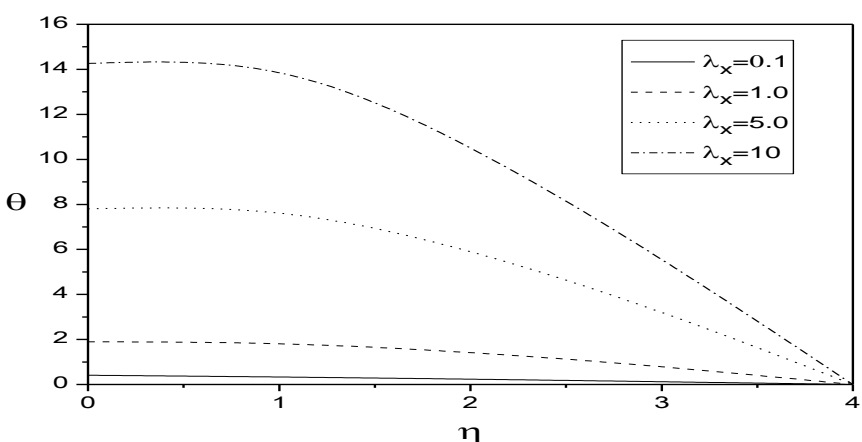

Fig.10 Variation of the temparature component $\theta$ with $\lambda_{x}$ for $G r=\gamma=K=M=0.1, R=1, \operatorname{Pr}=0.72$.

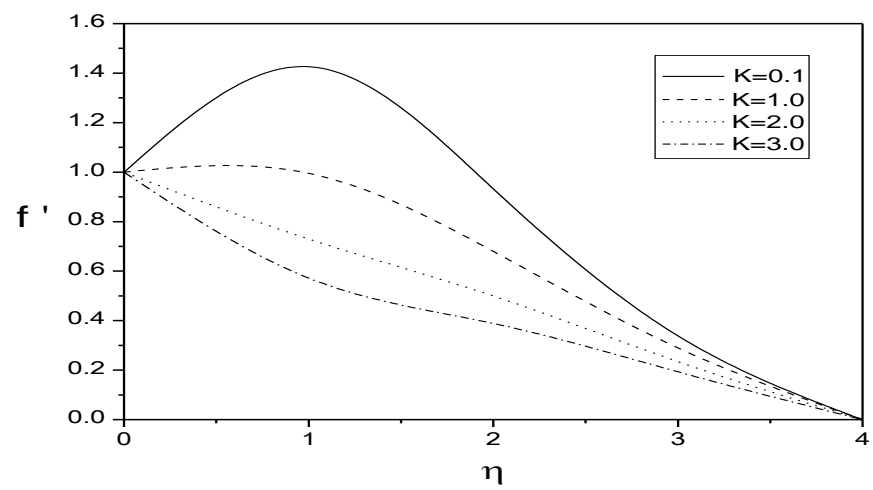

Fig.11: Variation of the velocity component $f^{\prime}$ with $K$ for $G r=\gamma=\lambda_{x}=M=0.1, R=1, \operatorname{Pr}=0.72$.

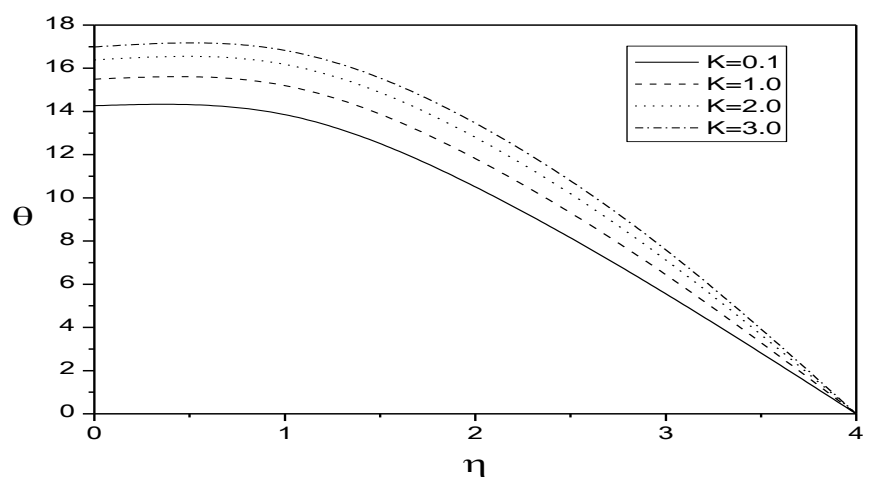

Fig.12: Variation of the temperature $\theta$ with $K$ for $G r=\gamma=\lambda_{x}=M=0.1, R=1, \operatorname{Pr}=0.72$. 


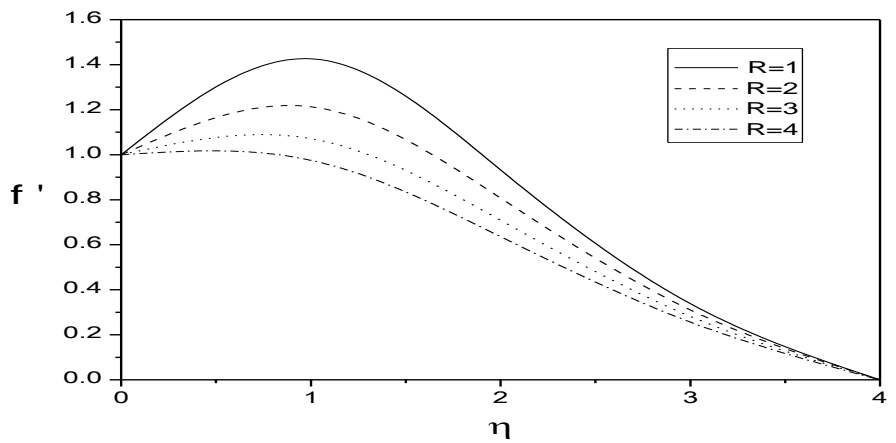

Fig.13: Variation of the velocity component $f^{\prime}$ with $\boldsymbol{R}$ for $G r=\gamma=\lambda_{x}=K=M=0.1, \operatorname{Pr}=0.72$.

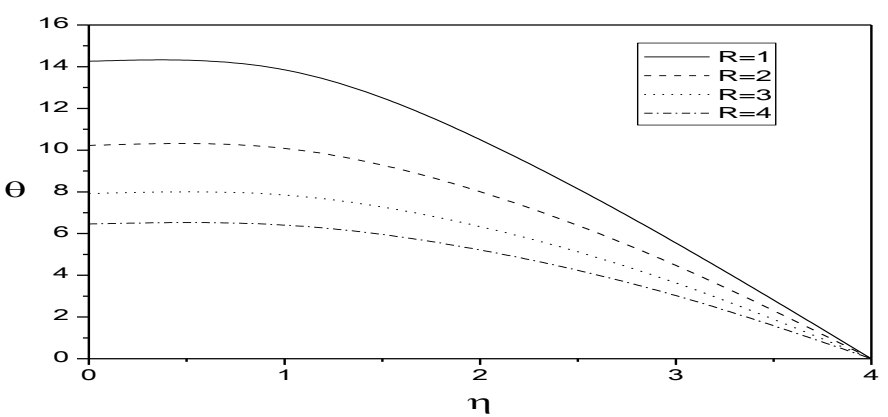

Fig.14: Variation of the temperature $\theta$ with $\boldsymbol{R}$ for $G r=\gamma=\lambda_{x}=K=M=0.1, \operatorname{Pr}=0.72$.

Table 1: Comparison of $f^{\prime \prime}(0), \theta^{\prime}(0), \theta(0)$ at the plate with $G r, \gamma, \lambda_{x}, P r$.

\begin{tabular}{|c|c|c|c|c|c|c|c|c|c|}
\hline \multirow[b]{2}{*}{$\gamma$} & \multirow[b]{2}{*}{ Gr } & \multirow[b]{2}{*}{$\operatorname{Pr}$} & \multirow[b]{2}{*}{$\lambda_{x}$} & \multicolumn{3}{|c|}{ Makinde (2011) } & \multicolumn{3}{|c|}{ Present work } \\
\hline & & & & $f^{\prime \prime}(0)$ & $\theta^{\prime}(0)$ & $\theta(0)$ & $f^{\prime \prime}(0)$ & $\theta^{\prime}(0)$ & $\theta(0)$ \\
\hline 0.1 & 0.1 & 0.72 & 1.0 & -0.200518 & 0.076578477 & 1.76578477 & -0.1928030 & 0.18023500 & 1.7802350 \\
\hline 1.0 & 0.1 & 0.72 & 1.0 & -0.2459676 & 0.281651449 & 1.28165144 & -0.2767380 & 0.71172200 & 1.2811720 \\
\hline 10 & 0.1 & 0.72 & 1.0 & -0.2695171 & 0.382952717 & 1.03829527 & -0.3243060 & 1.00535000 & 1.1005300 \\
\hline 0.1 & 0.5 & 0.72 & 1.0 & 0.4221216 & 0.048257030 & 1.48257030 & 0.8075150 & 0.14894500 & 1.4894500 \\
\hline 0.1 & 1.0 & 0.72 & 1.0 & 0.9895493 & 0.034011263 & 1.34011263 & 1.7720300 & 0.12691800 & 1.3426918 \\
\hline 0.1 & 0.1 & 3.00 & 1.0 & -0.3748695 & -0.023814576 & 0.76185423 & -0.3494040 & 0.05734740 & 0.7657347 \\
\hline 0.1 & 0.1 & 7.10 & 1.0 & -0.4138825 & -0.057164001 & 0.42835998 & -0.4266100 & -0.00937855 & 0.4206214 \\
\hline 0.1 & 0.1 & 0.72 & 5.0 & 0.3741286 & 0.576670381 & 6.76670381 & 0.7687850 & 1.08566000 & 6.8566000 \\
\hline 0.1 & 0.1 & 0.72 & 10 & 0.9010790 & 1.106605802 & 12.0660580 & 1.7006500 & 2.04775000 & 12.477500 \\
\hline
\end{tabular}

Table 2: Variation of $f^{\prime \prime}(0), \theta^{\prime}(0) \& \theta(0)$ at the plate with $K, M$ and $R$ for

\begin{tabular}{|c|c|c|c|c|c|}
\multicolumn{7}{|c|}{$G r=\gamma=0.1, \lambda_{x}=10, \operatorname{Pr}=0.72}$. \\
\hline $\boldsymbol{K}$ & $\boldsymbol{M}$ & $\boldsymbol{R}$ & $f^{\prime \prime}(0)$ & $\theta^{\prime}(0)$ & $\theta(0)$ \\
\hline 0.1 & 0.1 & 1 & 1.06254 & 1.32644 & 14.2644 \\
1 & 0.1 & 1 & 0.210499 & 1.44891 & 15.4891 \\
2 & 0.1 & 1 & -0.398815 & 1.53879 & 16.3879 \\
0.1 & 3 & 1 & -0.842902 & 1.59868 & 16.9868 \\
0.1 & 4 & 1 & -1.50081 & 1.6727 & 17.727 \\
0.1 & 0.1 & 2 & 0.635751 & 0.92254 & 10.2254 \\
0.1 & 0.1 & 3 & 0.37142 & 0.691855 & 7.91855 \\
\hline
\end{tabular}

